\title{
Existence criteria for positive solutions of a nonlinear difference inequality
}

\author{
by Sui Sun Cheng (Hsinchu) and Guang Zhang (Datong)
}

\begin{abstract}
This paper is concerned with a class of nonlinear difference inequalities which include many different classes of difference inequalities and equations as special cases. By means of a Riccati type transformation, necessary and sufficient conditions for the existence of eventually positive solutions and positive nonincreasing solutions are obtained. Various type of comparison theorems are also derived as applications, which extends many theorems in the literature.
\end{abstract}

1. Introduction. This paper is concerned with a class of delay difference inequalities of the form

$$
\begin{aligned}
\Delta x_{n}+a(n) x_{n}-b(n) \Delta x_{n-\sigma} & \\
& +\sum_{i=1}^{k} p_{i}(n) \prod_{j=1}^{m_{i}}\left|x_{n-\tau_{i j}(n)}\right|^{\alpha_{i j}} \operatorname{sgn} x_{n-\tau_{i j}(n)} \leq 0,
\end{aligned}
$$

where $\{a(n)\},\{b(n)\},\left\{p_{1}(n)\right\}, \ldots,\left\{p_{k}(n)\right\}$ are real sequences, $\alpha_{11}, \ldots, \alpha_{k m_{k}}$ are nonnegative numbers, and $\sigma, \tau_{11}(n), \ldots, \tau_{k m_{k}}(n)$ are nonnegative integers. Additional conditions related to these parameters will be given later. Here, we briefly explain the motivations behind our investigations.

First note that when $b(n) \equiv 0, m_{1}=\ldots=m_{k}=1$, and $\tau_{i 1}(n) \equiv \tau_{i}$ for $1 \leq i \leq k,(1.1)$ reduces to the linear difference inequality

$$
\Delta x_{n}+a(n) x_{n}+\sum_{i=1}^{k} p_{i}(n) x_{n-\tau_{i}} \leq 0, \quad n \geq 0,
$$

2000 Mathematics Subject Classification: Primary 39A10.

Key words and phrases: nonlinear delay difference inequality, $n$th order linear difference equation, neutral difference equation, eventually positive solution, eventually positive nonincreasing solution, Sturm type comparison theorem, limit comparison theorem.

Acknowledgments. The authors are indebted to Prof. J. Popenda for many valuable suggestions. 
two particular cases of which are the linear recurrence relation

$$
x_{n+1}+p_{0}(n) x_{n}+p_{1}(n) x_{n-1}+\ldots+p_{k}(n) x_{n-k}=0,
$$

and the second order difference equation

$$
\Delta^{2} x_{n-1}+q(n) x_{n}=0 .
$$

Such relations have been studied in some depth by a number of authors (see e.g. $[1,2,8,10])$. In particular, Yan and Qian in [8] study the difference equation

$$
\Delta x_{n}+\sum_{i=1}^{k} p_{i}(n) x_{n-\tau_{i}}=0
$$

and obtain several useful limit comparison theorems.

When $b(n) \equiv 0$, the relation (1.1) can be considered as a discrete analog of the first order nonlinear delay differential inequality of the form $[7,9]$

$$
y^{\prime}(t)+a(t) y(t)+\sum_{j=1}^{k} p_{j}(t) \prod_{i=1}^{m_{j}} \mid y\left(t-\left.\tau_{i j}(t)\right|^{\alpha_{i j}} \operatorname{sgn} y\left(t-\tau_{i j}(t)\right) \leq 0 .\right.
$$

A closely related equation [7] and particular cases of this inequality including

$$
y^{\prime}(t)+p(t) y(t-\tau(t))=0
$$

have been extensively studied [2] and a variety of oscillation and stability criteria have been demonstrated.

Next note that when $b(n) \equiv b, a(n) \equiv 0, m_{1}=\ldots=m_{k}=1$, and $\tau_{i 1}(n) \equiv \tau_{i}$ for $1 \leq i \leq k,(1.1)$ reduces to the linear difference inequality

$$
\Delta\left(x_{n}-b x_{n-\sigma}\right)+\sum_{i=1}^{k} p_{i}(n) x_{n-\tau_{i}} \leq 0, \quad n \geq 0 .
$$

Such a neutral type relation has also been studied by a number of authors, and related results can be found in $[4,5]$.

A particular case of (1.1) also arises when neutral difference equations of the form

$$
\Delta\left(y_{n}-p_{n} y_{n-\sigma}\right)+q_{n} y_{n-\tau}=0,
$$

are considered. More precisely, let us assume that such an equation has a solution $\left\{y_{n}\right\}$. Then the sequence $\left\{z_{n}\right\}$ defined by

$$
z_{n}=y_{n}-p_{n} y_{n-\sigma}
$$

will satisfy

$$
\Delta z_{n}+q_{n} y_{n-\tau}=\Delta z_{n}+q_{n} z_{n-\tau}+q_{n} p_{n-\tau} y_{n-\tau-\sigma}=0
$$

and

$$
\Delta z_{n-\sigma}+q_{n-\sigma} y_{n-\tau-\sigma}=0 .
$$


Thus $\left\{z_{n}\right\}$ is a solution of

$$
q_{n-\sigma} \Delta z_{n}-q_{n} p_{n-\tau} \Delta z_{n-\sigma}+q_{n} q_{n-\sigma} z_{n-\tau}=0,
$$

or

$$
\Delta z_{n}-\frac{q_{n} p_{n-\tau}}{q_{n-\sigma}} \Delta z_{n-\sigma}+q_{n} z_{n-\tau}=0
$$

in case $q_{n} \neq 0$ for all large $n$. The last equation is also a special case of (1.1).

Finally, note that discrete Emden-Fowler type equations of the form

$$
\Delta^{2} x_{n-1}+\sum_{i=1}^{k} p_{i}(n) x_{n-\tau_{i}}^{\alpha_{i}}=0
$$

are also special cases of our equation (1.1). A number of papers are devoted to such equations, see for example [6].

In view of the above discussion, we expect that a variety of information can be drawn by studying relation (1.1). In this paper, we will be concerned with the existence of positive and positive nonincreasing solutions of this relation. It turns out that if we are concerned with the existence of positive solutions, then it is more convenient to assume that $b(n) \equiv 0$; while if we are concerned with the existence of positive nonincreasing solutions, that $a(n) \equiv 0$. For this reason, we will study this relation in two separate parts, even though parallel developments will be presented. We remark that some of the notations will be repeated in both parts. Confusion should not arise, however, since both parts are self-contained. We also remark that if we are concerned with positive solutions, then there is no need to deal with (1.1), but to study the simpler relation

$$
\Delta x_{n}+a(n) x_{n}-b(n) \Delta x_{n-\sigma}+\sum_{i=1}^{k} p_{i}(n) \prod_{j=1}^{m_{i}} x_{n-\tau_{i j}(n)}^{\alpha_{i j}} \leq 0 .
$$

Indeed, this assertion follows from the fact that a positive sequence that satisfies (1.1) must also satisfy the simpler relation and vice versa. There are technical conveniences, however, if we are also concerned with a parallel development for the existence of negative or negative nondecreasing solutions of the dual relation of (1.1):

$$
\Delta x_{n}+a(n) x_{n}-b(n) \Delta x_{n-\sigma}+\sum_{i=1}^{k} p_{i}(n) \prod_{j=1}^{m_{i}}\left|x_{n-\tau_{i j}(n)}\right|^{\alpha_{i j}} \operatorname{sgn} x_{n-\tau_{i j}(n)} \geq 0,
$$

even though this relation may not allow the negative of a solution of (1.1) as its own solution. For this reason, we will keep the original form of (1.1), although the reader may disregard the absolute functions and sign functions in all the relations or equations that follow. 
For convenience, a real number $x$ will be called subnormal if $x \leq 1$ and strictly subnormal if $x<1$. Hence a real sequence $\left\{x_{k}\right\}$ is said to be eventually subnormal if $x_{k} \leq 1$ for all large $k$. A sequence $x=\left\{x_{k}\right\}_{n=a}^{\infty}$ is said to dominate the sequence $y=\left\{y_{k}\right\}_{n=a}^{\infty}$ if $x_{k} \geq y_{k}$ for $n \geq a$. As is customary, an empty product will be taken to be 1 .

\section{Part 1}

2.1. Assumptions. This part is concerned with the class of delay difference inequalities of the form

$$
\begin{aligned}
& \Delta x_{n}+a(n) x_{n} \\
& \quad+\sum_{i=1}^{k} p_{i}(n) \prod_{j=1}^{m_{i}}\left|x_{n-\tau_{i j}(n)}\right|^{\alpha_{i j}} \operatorname{sgn} x_{n-\tau_{i j}(n)} \leq 0, \quad n=0,1,2, \ldots,
\end{aligned}
$$

where

(H1) $\{a(n)\}_{n=0}^{\infty}$ is a real sequence,

(H2) $\left\{p_{1}(n)\right\}_{n=0}^{\infty}, \ldots,\left\{p_{k}(n)\right\}_{n=0}^{\infty}$ are nonnegative sequences,

(H3) $\alpha_{11}, \ldots, \alpha_{k m_{k}}$ are nonnegative numbers such that

$$
\sum_{j=1}^{m_{i}} \alpha_{i j}=1, \quad i=1, \ldots, k,
$$

(H4) $\tau_{11}(n), \ldots, \tau_{k m_{k}}(n)$ are nonnegative integers for $n \geq 0$ such that $\tau_{i j}(n) \leq n$ for $1 \leq i \leq k, 1 \leq j \leq m_{k}, n \geq 0$ and

$$
\lim _{n \rightarrow \infty}\left(n-\tau_{i j}(n)\right)=\infty, \quad 1 \leq i \leq k, 1 \leq j \leq m_{k},
$$

(H5) $\tau^{*}=\sup \left\{\tau_{i j}(n) \mid 1 \leq i \leq k, 1 \leq j \leq m_{k}, n \geq 0\right\}$ is a finite nonnegative integer.

A solution of (2.1) is a real sequence $\left\{x_{n}\right\}_{n=-\tau^{*}}^{\infty}$ which satisfies (2.1) for $n \geq 0$. Since $(2.1)$ can be written in the form $x_{n+1} \leq F\left(x_{n-\alpha(n)}, \ldots, x_{n-\beta(n)}\right)$, it is easy to formulate an existence theorem for this relation. We will be concerned with necessary and sufficient conditions for the existence of eventually positive solutions of (2.1). For this reason, we will also relax the definition of a solution to mean one that satisfies (2.1) for all large $n$, instead of for $n \geq-\tau^{*}$.

2.2. Necessary and sufficient conditions. Let $\left\{x_{k}\right\}$ be an eventually positive solution of (2.1). Then there is an integer $N \geq \tau^{*}$ such that $x_{n}>0$ for $n \geq N-\tau^{*}$. Define a sequence $\left\{w_{n}\right\}$ by

$$
w_{n}=-\frac{\Delta x_{n}}{x_{n}}, \quad n \geq N-\tau^{*}
$$


Then $\left\{w_{n}\right\}$ is strictly subnormal for $n \geq N-\tau^{*}$ :

$$
0<\frac{x_{n+1}}{x_{n}}=1-w_{n}, \quad n \geq N-\tau^{*},
$$

and

$$
\frac{x_{n}}{x_{n-\tau_{i j}(n)}}=\left(1-w_{n-1}\right) \ldots\left(1-w_{n-\tau_{i j}(n)}\right), \quad n \geq N .
$$

Substituting these equalities into (2.1), we obtain, for $n \geq N$,

$$
w_{n} \geq a(n)+\sum_{i=1}^{k} p_{i}(n) \prod_{j=1}^{m_{i}} \prod_{s=n-\tau_{i j}(n)}^{n-1} \frac{1}{\left(1-w_{s}\right)^{\alpha_{i j}}} .
$$

THEOREM 2.2.1. Suppose (2.1) has an eventually positive solution. Then (2.2) has an eventually strictly subnormal solution which eventually dominates $\{a(n)\}$. The converse also holds.

Proof. It suffices to directly verify that if $\left\{w_{n}\right\}$ is a solution of (2.2) which is strictly subnormal for $n \geq N-\tau^{*} \geq 0$ and dominates $\{a(n)\}$, then the sequence $\left\{x_{n}\right\}$ defined by $x_{N}=1$ and

$$
x_{n+1}=\prod_{i=N}^{n}\left(1-w_{i}\right), \quad n \geq N,
$$

is an eventually positive solution of (2.1).

Let us now formally define a sequence $\left\{w^{(t)}\right\}$ of sequences as follows: Let $N \geq \tau^{*}$ be a nonnegative integer. We define $w_{n}^{(0)}=a(n)$ for $n \geq 0$, and for $t=0,1,2, \ldots$, we inductively define

$$
w_{n}^{(t+1)}=a(n)+\sum_{i=1}^{k} p_{i}(n) \prod_{j=1}^{m_{i}} \prod_{s=n-\tau_{i j}(n)}^{n-1} \frac{1}{\left(1-w_{s}^{(t)}\right)^{\alpha_{i j}}}, \quad n \geq N
$$

and

$$
w_{n}^{(t+1)}=w_{n}^{(t)}, \quad n<N .
$$

For convenience, the sequence $\left\{w^{(t)}\right\}$ just defined will be called the companion sequence of (2.1) relative to the nonnegative integer $N$.

THEOREM 2.2.2. The relation (2.2) has a solution $\left\{w_{n}\right\}$ which is strictly subnormal and dominates $\{a(n)\}$ for $n \geq N-\tau^{*} \geq 0$ if, and only if, each term of the companion sequence $\left\{w^{(t)}\right\}$ of (2.1) relative to the integer $N$ is strictly subnormal for $n \geq N$, and $\left\{w^{(t)}\right\}$ converges pointwise to a sequence $\left\{u_{n}\right\}$ which is eventually strictly subnormal.

Proof. Let $\left\{w_{n}\right\}$ be a solution of $(2.2)$ such that $a(n) \leq w_{n}<1$ for $n \geq N-\tau^{*} \geq 0$. Then the companion sequence of (2.1) relative to the 
integer $N$ satisfies

$$
a(n)=w_{n}^{(0)} \leq w_{n}<1, \quad n \geq N-\tau^{*} .
$$

Therefore,

$$
\frac{1}{\left(1-w_{s}^{(0)}\right)^{\alpha_{i j}}} \leq \frac{1}{\left(1-w_{s}\right)^{\alpha_{i j}}}, \quad s \geq N-\tau^{*}
$$

so that

$$
\begin{aligned}
w_{n}^{(0)} & =a(n) \leq a(n)+\sum_{i=1}^{k} p_{i}(n) \prod_{j=1}^{m_{i}} \prod_{s=n-\tau_{i j}(n)}^{n-1} \frac{1}{\left(1-w_{s}^{(0)}\right)^{\alpha_{i j}}}=w_{n}^{(1)} \\
& \leq a(n)+\sum_{i=1}^{k} p_{i}(n) \prod_{j=1}^{m_{i}} \prod_{s=n-\tau_{i j}(n)}^{n-1} \frac{1}{\left(1-w_{s}\right)^{\alpha_{i j}}} \leq w_{n}, \quad n \geq N .
\end{aligned}
$$

Similarly, we have

$$
\begin{aligned}
w_{n}^{(1)} & =a(n)+\sum_{i=1}^{k} p_{i}(n) \prod_{j=1}^{m_{i}} \prod_{s=n-\tau_{i j}(n)}^{n-1} \frac{1}{\left(1-w_{s}^{(0)}\right)^{\alpha_{i j}}} \\
& \leq a(n)+\sum_{i=1}^{k} p_{i}(n) \prod_{j=1}^{m_{i}} \prod_{s=n-\tau_{i j}(n)}^{n-1} \frac{1}{\left(1-w_{s}^{(1)}\right)^{\alpha_{i j}}}=w_{n}^{(2)} \leq w_{n}
\end{aligned}
$$

for $n \geq N$. Inductively, we may show that $w_{n}^{(0)} \leq w_{n}^{(1)} \leq w_{n}^{(2)} \leq \ldots \leq w_{n}$ $<1$ for $n \geq N$.

Conversely, if each $w^{(t)}$ is strictly subnormal and $\left\{w^{(t)}\right\}$ is pointwise convergent to a strictly subnormal sequence $\left\{u_{n}\right\}$ for $n \geq N$, then as before, we may show that $w_{n}^{(0)} \leq w_{n}^{(1)} \leq w_{n}^{(2)} \leq \ldots \leq u_{n}<1$ for $n \geq N$. By the Lebesgue dominated convergence theorem, we may take limits on both sides of (2.4) as $t$ tends to infinity to obtain

$$
u_{n}=a(n)+\sum_{i=1}^{k} p_{i}(n) \prod_{j=1}^{m_{i}} \prod_{s=n-\tau_{i j}(n)}^{n-1} \frac{1}{\left(1-u_{s}\right)^{\alpha_{i j}}}, \quad n \geq N .
$$

The proof is complete.

We pause to make two important observations here. Note first that the companion sequence $\left\{w^{(t)}\right\}$ is nondecreasing in $t$ (so that $a(n) \leq w_{n}^{(t)} \leq$ $w_{n}^{(t+1)}$ for $\left.n \geq N\right)$ provided each $w^{(t)}$ is strictly subnormal. We will refer to this property as the monotone nature of the companion sequence $\left\{w^{(t)}\right\}$. Note further that the sequence $\left\{u_{n}\right\}$ in the above theorem satisfies (2.6), which is a system of equalities (cf. (2.2)). Since we may easily verify that the sequence $\left\{x_{n}\right\}$ defined by $x_{N}=1$ and (2.3) is an eventually positive 
solution of

$$
\Delta x_{n}+a(n) x_{n}+\sum_{i=1}^{k} p_{i}(n) \prod_{j=1}^{m_{i}}\left|x_{n-\tau_{i j}(n)}\right|^{\alpha_{i j}} \operatorname{sgn} x_{n-\tau_{i j}(n)}=0, \quad n \geq 0
$$

and since every solution of (2.7) is also a solution of (2.1), we see that the following result must hold.

THEOREM 2.2.3. The relation (2.1) has an eventually positive solution if, and only if, (2.7) has an eventually positive solution.

In case $a(n) \equiv a, p_{i}(n) \equiv p_{i}>0$ for $1 \leq i \leq k$, and $\tau_{i j}(n) \equiv \tau_{i j}$ for $1 \leq i \leq k$ and $1 \leq j \leq m_{i}$, the relation (2.1) reduces to

$$
\Delta x_{n}+a x_{n}+\sum_{i=1}^{k} p_{i} \prod_{j=1}^{m_{i}}\left|x_{n-\tau_{i j}}\right|^{\alpha_{i j}} \operatorname{sgn} x_{n-\tau_{i j}} \leq 0, \quad n \geq 0,
$$

and for large $N$, the sequence $\left\{w_{n}^{(0)}\right\}_{n=N}^{\infty}$ becomes the constant sequence $\{a\}$, and the sequence $\left\{w_{n}^{(1)}\right\}_{n=N}^{\infty}$ becomes the constant sequence $\left\{w^{(1)}\right\}$ defined by $w^{(1)}=a+\lambda_{1}$, where

$$
\lambda_{1}=\sum_{i=1}^{k} p_{i}(1-a)^{-\sum_{j=1}^{m_{i}} \alpha_{i j} \tau_{i j}}
$$

Inductively, we see that if we define $\lambda_{0}=0$ and

$$
\lambda_{t+1}=\sum_{i=1}^{k} p_{i}\left(1-a-\lambda_{t}\right)^{-\sum_{j=1}^{m_{i}} \alpha_{i j} \tau_{i j}}, \quad t=0,1,2, \ldots,
$$

then the sequence $\left\{w_{n}^{(t)}\right\}_{n=N}^{\infty}$ is equal to the constant sequence $\left\{a+\lambda_{t}\right\}$ for each $t \geq 1$. If (2.8) has an eventually positive solution, then by Theorem 2.2.2, $a+\lambda_{t}<1$ for $t=0,1,2, \ldots, 0<\lambda_{1} \leq \lambda_{2} \leq \ldots$, and $\left\{\lambda_{t}\right\}$ converges to a number in $(0,1-a)$. The converse clearly holds.

THEOREM 2.2.4. The relation (2.8) has an eventually positive solution if , and only if, $a<1$ and the sequence $\left\{\lambda_{t}\right\}$ defined by $\lambda_{0}=0$ and (2.9) satisfies

$$
a+\lambda_{1} \leq a+\lambda_{2} \leq \ldots<1
$$

and converges to a number in $(0,1-a)$.

Therefore if (2.8) has an eventually positive solution, then by taking limits on both sides of (2.9), we see that the associated "characteristic equation"

$$
\lambda=\sum_{i=1}^{k} p_{i}(1-a-\lambda)^{-\sum_{j=1}^{m_{i}} \alpha_{i j} \tau_{i j}}
$$


has a real root $\lambda=\lambda^{*}$ in $(0,1-a)$. Conversely, if $(2.10)$ has a real root $\lambda=\lambda^{*}$ in $(0,1-a)$, then $a<1$ so that $\lambda_{1}>0$. Furthermore,

$$
0<\lambda_{1}=\sum_{i=1}^{k} p_{i}(1-a)^{-\sum_{j=1}^{m_{i}} \alpha_{i j} \tau_{i j}} \leq \sum_{i=1}^{k} p_{i}\left(1-a-\lambda^{*}\right)^{-\sum_{j=1}^{m_{i}} \alpha_{i j} \tau_{i j}}=\lambda^{*},
$$

and inductively, $\lambda_{t}<\lambda^{*}$ for all $t \geq 2$. By the monotone nature of the sequence $\left\{w^{(t)}\right\}$, we see that $\left\{\lambda_{t}\right\}$ is also monotone and hence it converges to a number in $\left(0, \lambda^{*}\right]$. Thus, $(2.8)$ has an eventually positive solution by Theorem 2.2.2.

To summarize, (2.8) has an eventually positive solution if, and only if, the characteristic equation (2.10) has a real root in $(0,1-a)$.

2.3. Consequences. As an immediate corollary of Theorem 2.2.1, we seek a constant solution $\{w\}$ of $(2.2)$ and come up with the following result.

THEOREM 2.3.1. If there is a number $w<1$ and an integer $N$ such that

$$
w \geq a(n)+\sum_{i=1}^{k} p_{i}(n)(1-w)^{-\sum_{j=1}^{m_{i}} \alpha_{i j} \tau_{i j}(n)}, \quad n \geq N,
$$

then (2.1) has an eventually positive solution.

Therefore, if $a(n) \equiv 0$, and

$$
\sum_{j=1}^{m_{i}} \alpha_{i j} \tau_{i j}(n) \equiv \tau>0, \quad 1 \leq i \leq k,
$$

then (2.1) will have an eventually positive solution provided that

$$
\sum_{i=1}^{k} p_{i}(n) \leq \frac{\tau^{\tau}}{(1+\tau)^{\tau+1}}, \quad n \geq N
$$

Indeed, (2.11) will be satisfied if

$$
\sum_{i=1}^{k} p(n) \leq \max _{0<w<1} w(1-w)^{\tau}=\frac{\tau^{\tau}}{(1+\tau)^{\tau+1}}
$$

which leads to the fact that $\{1 /(1+\tau)\}$ is a strictly subnormal constant solution of (2.11).

As an immediate corollary of Theorems 2.2.1 and 2.2.2, we have the following result.

THEOREM 2.3.2. The relation (2.1) cannot have an eventually positive solution if for any integer $N \geq \tau^{*}$, there is some integer $n \geq N$ such that the companion sequence of $(2.1)$ relative to $N$ satisfies $w_{n}^{(t)} \geq 1$ for some $t \geq 0$. 
Therefore, if for any $N \geq \tau^{*}$, there is some integer $n \geq N$ such that $w_{n}^{(0)}=a(n) \geq 1$, or

$$
w_{n}^{(1)}=a(n)+\sum_{i=1}^{k} p_{i}(n) \prod_{j=1}^{m_{i}} \prod_{i=n-\tau_{i j}(n)}^{n-1} \frac{1}{(1-a(i))^{\alpha_{i j}}} \geq 1,
$$

then (2.1) cannot have an eventually positive solution.

Next, we find more sophisticated conditions under which (2.1) cannot have an eventually positive solution.

TheOREM 2.3.3. Suppose there is an integer $N \geq \tau^{*}$ and there are constants $\tau$ and $a_{*}$ such that

$$
\begin{gathered}
\inf _{n \geq N-\tau^{*}} a(n) \geq a_{*}>-\infty, \\
0 \leq \sum_{j=1}^{m_{i}} \alpha_{i j} \tau_{i j}(n) \leq \tau, \quad n \geq N-\tau^{*}>0,1 \leq i \leq k,
\end{gathered}
$$

and

$$
\inf _{n \geq N-\tau^{*}, 0<\mu<1-a_{*}}\left\{\frac{1}{\mu} \sum_{i=1}^{k} p_{i}(n)\left(1-a_{*}-\mu\right)^{-\sum_{j=1}^{m_{i}} \alpha_{i j} \tau_{i j}(n)}\right\}>1,
$$

then (2.1) cannot have an eventually positive solution.

P r o of. Let $\left\{w^{(t)}\right\}$ be the companion sequence of (2.1). In view of Theorem 2.3.2 and the monotone nature of $\left\{w^{(t)}\right\}$, we may assume without loss of generality that each $w^{(t)}$ dominates $\{a(n)\}$ and is strictly subnormal. Note that if $a_{*} \geq 1$, then $w_{n}^{(0)} \geq a(n) \geq a_{*} \geq 1$, which implies (see Theorem 2.2.2) that the nondecreasing sequence $\left\{w^{(t)}\right\}$ cannot converge to a strictly subnormal limiting sequence and hence our assertion is true.

Next, note that $w_{n}^{(0)}=a(n) \geq a_{*}$ for $n \geq N-\tau^{*}$, and

$$
\begin{aligned}
w_{n}^{(1)} & =a(n)+\sum_{i=1}^{k} p_{i}(n) \prod_{j=1}^{m_{i}} \prod_{s=n-\tau_{i j}(n)}^{n-1} \frac{1}{\left(1-w_{s}^{(0)}\right)^{\alpha_{i j}}} \\
& \geq a(n)+\sum_{i=1}^{k} p_{i}(n) \prod_{j=1}^{m_{i}} \prod_{s=n-\tau_{i j}(n)}^{n-1} \frac{1}{\left(1-a_{*}\right)^{\alpha_{i j}}} \\
& =a(n)+\sum_{i=1}^{k} p_{i}(n)\left(1-a_{*}\right)^{-\sum_{j=1}^{m_{i}} \alpha_{i j} \tau_{i j}(n)} \\
& \geq a(n)+\mu_{1}
\end{aligned}
$$

for $n \geq N$, and

$$
w_{n}^{(1)} \geq a_{*}+\mu_{1}
$$


for $n \geq N-\tau^{*}$, where

$$
\mu_{1}=\inf _{n \geq N-\tau^{*}} \sum_{i=1}^{k} p_{i}(n)\left(1-a_{*}\right)^{-\sum_{j=1}^{m_{i}} \alpha_{i j} \tau_{i j}(n)} .
$$

If $a_{*}+\mu_{1} \geq 1$, then Theorem 2.3.2 again implies the truth of our assertion. Inductively, if we define

$$
\mu_{t+1}=\inf _{n \geq N-\tau^{*}} \sum_{i=1}^{k} p_{i}(n)\left(1-a_{*}-\mu_{t}\right)^{-\sum_{j=1}^{m_{i}} \alpha_{i j} \tau_{i j}(n)}, \quad t=1,2, \ldots,
$$

then

$$
w_{n}^{(t)} \geq a(n)+\mu_{t}, \quad n \geq N, t \geq 1,
$$

and

$$
w_{n}^{(t)} \geq a^{*}+\mu_{t}, \quad n \geq N-\tau^{*}, t \geq 1 .
$$

Similarly, (2.15) shows that if $a_{*}+\mu_{t} \geq 1$ for any $t \geq 1$, then our assertion is true by Theorem 2.3.2.

Assume that $a_{*}<1$ and $a_{*}+\mu_{t}<1$ for $t \geq 1$. We assert that $0<\mu_{1} \leq$ $\mu_{2} \leq \ldots \leq 1-a_{*}$. Indeed, note first that $\mu_{1} \geq 0$. If $\mu_{1}=0$, then there is a sequence of integers $\left\{n_{s}\right\}$ such that

$$
\lim _{s \rightarrow \infty} \sum_{i=1}^{k} p_{i}\left(n_{s}\right)\left(1-a_{*}\right)^{-\sum_{j=1}^{m_{i}} \alpha_{i j} \tau_{i j}\left(n_{s}\right)}=0 .
$$

Take $\mu^{\prime}=\left(1-a_{*}\right) / 2$, then $0<\mu^{\prime}<1-a_{*}$ and

$$
\begin{aligned}
\lim _{s \rightarrow \infty} \frac{1}{\mu^{\prime}} \sum_{i=1}^{k} p_{i}\left(n_{s}\right)(1 & \left.-a_{*}-\mu^{\prime}\right)^{-\sum_{j=1}^{m_{i}} \alpha_{i j} \tau_{i j}\left(n_{s}\right)} \\
& \leq \lim _{s \rightarrow \infty} \frac{2^{\tau}}{\mu^{\prime}} \sum_{i=1}^{k} p_{i}\left(n_{s}\right)\left(1-a_{*}\right)^{-\sum_{j=1}^{m_{i}} \alpha_{i j} \tau_{i j}\left(n_{s}\right)}=0,
\end{aligned}
$$

contrary to (2.13). Since $\mu_{1}>0$, we have

$$
\begin{aligned}
\mu_{2} & =\inf _{n \geq N-\tau^{*}} \sum_{i=1}^{k} p_{i}(n)\left(1-a_{*}-\mu_{1}\right)^{-\sum_{j=1}^{m_{i}} \alpha_{i j} \tau_{i j}(n)} \\
& \geq \inf _{n \geq N-\tau^{*}} \sum_{i=1}^{k} p_{i}(n)\left(1-a_{*}\right)^{-\sum_{j=1}^{m_{i}} \alpha_{i j} \tau_{i j}(n)}=\mu_{1} .
\end{aligned}
$$

Inductively, we see that $0<\mu_{1} \leq \mu_{2} \leq \ldots \leq 1-a_{*}$ as required. Let $\mu^{*}=\lim _{t \rightarrow \infty} \mu_{t}$. If $\mu^{*}<1-a_{*}$, then in view of (2.14), we have

$$
\mu^{*}=\inf _{n \geq N-\tau^{*}} \sum_{i=1}^{k} p_{i}(n)\left(1-a_{*}-\mu^{*}\right)^{-\sum_{j=1}^{m_{i}} \alpha_{i j} \tau_{i j}(n)},
$$


contrary to (2.13). Hence $\mu^{*}=1-a_{*}$. But then by (2.15), we see that $w_{n}^{(\infty)} \geq a(n)+1-a_{*} \geq 1$. This shows, in view of Theorem 2.2.2, that (2.1) cannot have an eventually positive solution. The proof is complete.

Note that for each fixed $n \geq N-\tau^{*}$,

$$
\inf _{0<\mu<1-a_{*}}\left\{\frac{1}{\mu} p_{i}(n)\left(1-a_{*}-\mu\right)^{-\sum_{j=1}^{m_{i}} \alpha_{i j} \tau_{i j}(n)}\right\}
$$

is attained at

$$
\mu_{i}=\left(1-a_{*}\right)\left(1+\sum_{j=1}^{m_{i}} \alpha_{i j} \tau_{i j}(n)\right)^{-1}
$$

and is equal to

$$
\frac{p_{i}(n)\left(1+\tau_{i}(n)\right)^{1+\tau_{i}(n)}}{\left(1-a_{*}\right)^{1+\tau_{i}(n)}\left(\tau_{i}(n)\right)^{\tau_{i}(n)}}
$$

where

$$
\tau_{i}(n)=\sum_{j=1}^{m_{i}} \alpha_{i j} \tau_{i j}(n)<\infty, \quad 1 \leq i \leq k, n \geq 0 .
$$

Therefore, if

$$
\liminf _{n \rightarrow \infty} \sum_{i=1}^{k} \frac{p_{i}(n)\left(1+\tau_{i}(n)\right)^{1+\tau_{i}(n)}}{\left(1-a_{*}\right)^{1+\tau_{i}(n)}\left(\tau_{i}(n)\right)^{\tau_{i}(n)}}>1,
$$

then, by Theorem 2.3.3, (2.1) cannot have an eventually positive solution. In particular, when $a(n) \equiv 0$ and $\tau_{i}(n) \equiv \tau$ for $1 \leq i \leq k$, the condition (2.17) is equivalent to

$$
\liminf _{n \rightarrow \infty} \sum_{i=1}^{k} p_{i}(n)>\frac{\tau^{\tau}}{(1+\tau)^{1+\tau}} .
$$

This shows that condition (2.12) is sharp.

Next, note that the arithmetic mean-geometric mean inequality implies

$$
\begin{aligned}
\frac{1}{\mu} \sum_{i=1}^{k} p_{i}(n)\left(1-a_{*}-\right. & \mu)^{-\tau_{i}(n)} \\
& \geq \frac{k}{\mu}\left\{\prod_{i=1}^{k} p_{i}(n)\right\}^{1 / k}\left\{\prod_{i=1}^{k}\left(1-a_{*}-\mu\right)^{-\tau_{i}(n)}\right\}^{1 / k} \\
& =k\left\{\prod_{i=1}^{k} p_{i}(n)\right\}^{1 / k}\left\{\prod_{i=1}^{k} \frac{1}{\mu\left(1-a_{*}-\mu\right)^{\tau_{i}(n)}}\right\}^{1 / k},
\end{aligned}
$$


where $\tau_{i}(n)$ is defined by $(2.16)$. Since

$$
\inf _{0<\mu<1-a_{*}} \frac{1}{\mu\left(1-a_{*}-\mu\right)^{\tau_{i}(n)}}=\frac{1}{\left(1-a_{*}\right)^{2}} \cdot \frac{\left(\tau_{i}(n)+1\right)^{\tau_{i}(n)+1}}{\tau_{i}(n)^{\tau_{i}(n)}},
$$

it follows that if

$$
\liminf _{n \rightarrow \infty} k\left\{\prod_{i=1}^{k} p_{i}(n)\right\}^{1 / k}\left\{\prod_{i=1}^{k} \frac{1}{\left(1-a_{*}\right)^{2}} \frac{\left(\tau_{i}(n)+1\right)^{\tau_{i}(n)+1}}{\tau_{i}(n)^{\tau_{i}(n)}}\right\}^{1 / k}>1,
$$

then, by Theorem 2.3.3, (2.1) cannot have an eventually positive solution.

Next, we find conditions under which the companion sequence $\left\{w^{(t)}\right\}$ of (2.1) is pointwise convergent to a strictly subnormal sequence.

Theorem 2.3.4. Suppose $\sup _{n \geq N-\tau^{*}} a(n)=a^{*}<1$ such that

$$
\sup _{n \geq N-\tau^{*}} \sum_{i=1}^{k} p_{i}(n)\left(1-a_{*}\right)^{-\sum_{j=1}^{m_{i}} \alpha_{i j} \tau_{i j}(n)}>0,
$$

and suppose further that there is a constant $\psi \in\left(0,1-a^{*}\right)$ such that

$$
\sup _{n \geq N-\tau^{*}}\left\{\frac{1}{\psi} \sum_{i=1}^{k} p_{i}(n)\left(1-a^{*}-\psi\right)^{-\sum_{j=1}^{m_{i}} \alpha_{i j} \tau_{i j}(n)}\right\} \leq 1 .
$$

Then (2.1) has an eventually positive solution.

Proof. Let $\left\{w^{(t)}\right\}$ be the companion sequence of (2.1). Note that

$$
w_{n}^{(1)}=a(n)+\sum_{i=1}^{k} p_{i}(n) \prod_{j=1}^{m_{i}} \prod_{s=n-\tau_{i j}(n)}^{n-1} \frac{1}{\left(1-w_{s}^{(0)}\right)^{\alpha_{i j}}} \leq a(n)+\psi_{1}
$$

for $n \geq N$, and

$$
w_{n}^{(1)} \leq a^{*}+\psi_{1}
$$

for $n \geq N-\tau^{*}$, where

$$
\psi_{1}=\sup _{n \geq N-\tau^{*}} \sum_{i=1}^{k} p_{i}(n)\left(1-a^{*}\right)^{-\sum_{j=1}^{m_{i}} \alpha_{i j} \tau_{i j}(n)} .
$$

By our assumptions,

$$
0<\psi_{1} \leq \sup _{n \geq N-\tau^{*}} \sum_{i=1}^{k} p_{i}(n)\left(1-a^{*}-\psi\right)^{-\sum_{j=1}^{m_{i}} \alpha_{i j} \tau_{i j}(n)} \leq \psi .
$$

Inductively, if we define

(2.19) $\quad \psi_{t+1}=\sup _{n \geq N-\tau^{*}} \sum_{i=1}^{k} p_{i}(n)\left(1-a^{*}-\psi_{t}\right)^{-\sum_{j=1}^{m_{i}} \alpha_{i j} \tau_{i j}(n)}, \quad t=1,2, \ldots$, 
then

$$
w_{n}^{(t)} \leq a(n)+\psi_{t}, \quad n \geq N, \quad t \geq 1
$$

and

$$
w_{n}^{(t)} \leq a^{*}+\psi_{t}, \quad n \geq N-\tau^{*}, \quad t \geq 1 .
$$

Under the condition (2.18), it is easily verified that $0<\psi_{1} \leq \psi_{2} \leq \ldots \leq \psi$. Thus, in view of (2.20), we see that the companion sequence of (2.1) satisfies $w_{n}^{(t)} \leq a^{*}+\psi_{t} \leq a^{*}+\psi$, which implies $w_{n}^{(\infty)} \leq a^{*}+\psi<1$ (since $\psi \in$ $\left.\left(0,1-a^{*}\right)\right)$ for $n \geq N$. The assertion now follows from Theorem 2.2.2.

As further applications of the previous results, we will derive a comparison theorem for relation (2.1). Let us consider a majorant inequality of the form

$$
\begin{aligned}
\Delta y_{n}+ & A(n) y_{n} \\
& +\sum_{i=1}^{k} P_{i}(n) \prod_{j=1}^{m_{i}}\left|y_{n-\sigma_{i j}(n)}\right|^{\alpha_{i j}} \operatorname{sgn} y_{n-\sigma_{i j}(n)} \leq 0, \quad n \geq 0,
\end{aligned}
$$

where $\{A(n)\}_{n=0}^{\infty}$ is a real sequence, $\left\{P_{1}(n)\right\}_{n=0}^{\infty}, \ldots,\left\{P_{k}(n)\right\}_{n=0}^{\infty}$ are nonnegative sequences, $\sigma_{11}(n), \ldots, \sigma_{k m_{k}}(n)$ are nonnegative integers which satisfy conditions similar to those satisfied by $\tau_{i j}(n)$, and the other parameters or parameter sequences are the same as those in (2.1). Also let the sequence $\left\{W^{(t)}\right\}$ of sequences be the companion sequence of $(2.21)$ relative to $N \geq \tau^{*}$ : $W_{n}^{(0)}=A(n)$ for $n \geq 0$, and for $t=0,1,2, \ldots$,

$$
W_{n}^{(t+1)}=A(n)+\sum_{i=1}^{k} P_{i}(n) \prod_{j=1}^{m_{i}} \prod_{s=n-\sigma_{i j}(n)}^{n-1} \frac{1}{\left(1-W_{s}^{(t)}\right)^{\alpha_{i j}}}, \quad n \geq N
$$

and $W_{n}^{(t+1)}=W_{n}^{(t)}$ for $n<N$.

TheOREM 2.3.5. Suppose $a(n) \leq A(n), \tau_{i j}(n)=\sigma_{i j}(n)$ and $p_{i}(n) \leq$ $P_{i}(n)$ for $1 \leq i \leq k, 1 \leq j \leq m_{k}$ and $n \geq 0$. If (2.21) has an eventually positive solution, then so does (2.1).

Indeed, if (2.21) has an eventually positive solution, then the relation

$$
W_{n} \geq A(n)+\sum_{i=1}^{k} P_{i}(n) \prod_{j=1}^{m_{i}} \prod_{s=n-\sigma_{i j}(n)}^{n-1} \frac{1}{\left(1-W_{s}\right)^{\alpha_{i j}}}
$$

has an eventually strictly subnormal solution $\left\{W_{n}\right\}$. Since 


$$
\begin{aligned}
W_{n} & \geq A(n)+\sum_{i=1}^{k} P_{i}(n) \prod_{j=1}^{m_{i}} \prod_{s=n-\sigma_{i j}(n)}^{n-1} \frac{1}{\left(1-W_{s}\right)^{\alpha_{i j}}} \\
& \geq a(n)+\sum_{i=1}^{k} p_{i}(n) \prod_{j=1}^{m_{i}} \prod_{s=n-\sigma_{i j}(n)}^{n-1} \frac{1}{\left(1-W_{s}\right)^{\alpha_{i j}}},
\end{aligned}
$$

we see that $\left\{W_{n}\right\}$ is also an eventually subnormal solution of (2.2). Our assertion now follows from Theorem 2.2.1 (cf. [8, Theorem 2], see also [3]).

Finally, we remark that if the condition $\tau_{i j}(n)=\sigma_{i j}(n)$ in the above theorem is changed to $\tau_{i j}(n) \leq \sigma_{i j}(n)$, then under the additional condition that (2.21) has an eventually positive and eventually nonincreasing solution, (2.1) will have an eventually positive solution. This is due to the fact that (2.22) will have an eventually strictly subnormal and eventually nonnegative solution $\left\{W_{n}\right\}$, which implies

$$
W_{n} \geq a(n)+\sum_{i=1}^{k} p_{i}(n) \prod_{j=1}^{m_{i}} \prod_{s=n-\tau_{i j}(n)}^{n-1} \frac{1}{\left(1-W_{s}\right)^{\alpha_{i j}}}
$$

\section{Part 2}

3.1. Assumptions. This part is concerned with the class of delay difference inequalities of the form

$$
\begin{aligned}
& \Delta x_{n}-b(n) \Delta x_{n-\sigma} \\
& \quad+\sum_{i=1}^{k} p_{i}(n) \prod_{j=1}^{m_{i}}\left|x_{n-\tau_{i j}(n)}\right|^{\alpha_{i j}} \operatorname{sgn} x_{n-\tau_{i j}(n)} \leq 0, \quad n \geq 0,
\end{aligned}
$$

where

(H0) $\sigma$ is a positive integer,

(H1) $\{b(n)\}_{n=0}^{\infty}$ is a nonnegative sequence,

(H2) $\left\{p_{1}(n)\right\}_{n=0}^{\infty}, \ldots,\left\{p_{k}(n)\right\}_{n=0}^{\infty}$ are nonnegative sequences,

(H3) $\alpha_{11}, \ldots, \alpha_{k m_{k}}$ are nonnegative numbers such that

$$
\sum_{j=1}^{m_{i}} \alpha_{i j}=1, \quad i=1, \ldots, k
$$

(H4) $\tau_{11}(n), \ldots, \tau_{k m_{k}}(n)$ are nonnegative integers for $n \geq 0$ such that $\tau_{i j}(n) \leq n$ for $1 \leq i \leq k, 1 \leq j \leq m_{k}, n \geq 0$ and

$$
\lim _{n \rightarrow \infty}\left(n-\tau_{i j}(n)\right)=\infty, \quad 1 \leq i \leq k, 1 \leq j \leq m_{k},
$$

(H5) $\tau^{*}=\max \left\{\sigma,\left\{\tau_{i j}(n) \mid 1 \leq i \leq k, 1 \leq j \leq m_{k}, n \geq 0\right\}\right\}$ is a finite nonnegative integer. 
A solution of (3.1) is a real sequence $\left\{x_{n}\right\}_{n=-\tau^{*}}^{\infty}$ which satisfies (3.1) for $n \geq 0$. Since (3.1) can be written in the form $x_{n+1} \leq F\left(x_{n-\alpha(n)}, \ldots, x_{n-\beta(n)}\right)$, it is easy to formulate an existence theorem for this relation. We will be concerned with necessary and sufficient conditions for the existence of eventually positive nonincreasing solutions of (3.1). For this reason, we will also relax the definition of a solution to mean one that satisfies (3.1) for all large $n$, instead of for $n \geq-\tau^{*}$.

3.2. Necessary and sufficient conditions. Let $\left\{x_{k}\right\}$ be an eventually positive solution of (3.1). Then there is an integer $N \geq \tau^{*}$ such that $x_{n}>0$ for $n \geq N-\tau^{*}$. Define a sequence $\left\{w_{n}\right\}$ by

$$
w_{n}=-\frac{\Delta x_{n}}{x_{n}}, \quad n \geq N-\tau^{*} .
$$

Then $\left\{w_{n}\right\}_{n=N}^{\infty}$ is strictly subnormal for $n \geq N-\tau^{*}$ :

and

$$
0<\frac{x_{n+1}}{x_{n}}=1-w_{n}, \quad n \geq N-\tau^{*},
$$

$$
\begin{aligned}
\frac{\Delta x_{n-\sigma}}{x_{n}} & =-w_{n-\sigma}\left(1-w_{n-\sigma}\right)^{-1} \ldots\left(1-w_{n-1}\right)^{-1}, \quad n \geq N, \\
\frac{x_{n}}{x_{n-\tau_{i j}(n)}} & =\left(1-w_{n-1}\right) \ldots\left(1-w_{n-\tau_{i j}(n)}\right), \quad n \geq N .
\end{aligned}
$$

Substituting these equalities into (3.1), we obtain

$$
w_{n} \geq b(n) w_{n-\sigma} \prod_{t=n-\sigma}^{n-1} \frac{1}{1-w_{t}}+\sum_{i=1}^{k} p_{i}(n) \prod_{j=1}^{m_{i}} \prod_{s=n-\tau_{i j}(n)}^{n-1} \frac{1}{\left(1-w_{s}\right)^{\alpha_{i j}}}
$$

for $n \geq N$.

THEOREM 3.2.1. The relation (3.1) has an eventually positive nonincreasing solution if, and only if, (3.2) has an eventually strictly subnormal and eventually nonnegative solution.

Proof. It suffices to verify directly that if $\left\{w_{n}\right\}$ is a solution of (3.2) which is strictly subnormal and eventually nonnegative for $n \geq N$, then the sequence $\left\{x_{n}\right\}$ defined by $x_{N}=1$ and

$$
x_{n+1}=\prod_{i=N}^{n}\left(1-w_{i}\right), \quad n \geq N,
$$

is an eventually positive solution of (3.1) which satisfies

$$
\Delta x_{n}=-w_{n} \prod_{i=N}^{n-1}\left(1-w_{i}\right) \leq 0 .
$$


We remark that in the proof of Theorem 2.2.1, the inequality $\Delta x_{n} \leq 0$ does not hold in general since $\left\{w_{n}\right\}$ is not nonnegative. This is one of the reasons for dealing with (1.1) in two parts.

Let us now formally define a sequence $\left\{w^{(t)}\right\}$ of sequences as follows: Let $N \geq \tau^{*}$. We define $w_{n}^{(0)}=0$ for $n \geq 0$; and for $t=0,1,2, \ldots$, we inductively define

$$
\begin{aligned}
w_{n}^{(t+1)}= & b(n) w_{n-\sigma}^{(t)} \prod_{i=n-\sigma}^{n-1} \frac{1}{1-w_{i}^{(t)}} \\
& +\sum_{i=1}^{k} p_{i}(n) \prod_{j=1}^{m_{i}} \prod_{s=n-\tau_{i j}(n)}^{n-1} \frac{1}{\left(1-w_{s}^{(t)}\right)^{\alpha_{i j}}}
\end{aligned}
$$

for $n \geq N$, and $w_{n}^{(t+1)}=0$ for $n<N$. For convenience, the sequence $\left\{w^{(t)}\right\}$ just defined will be called the companion sequence of (3.1) relative to the integer $N$.

THEOREM 3.2.2. The relation (3.2) has a solution $\left\{w_{n}\right\}$ which is nonnegative and strictly subnormal for $n \geq N-\tau^{*} \geq 0$ if, and only if, each $w^{(t)}$ of the companion sequence $\left\{w^{(t)}\right\}$ of $(3.1)$ relative to $N$ is strictly subnormal for $n \geq N$, and $\left\{w^{(t)}\right\}$ is pointwise convergent to a sequence $\left\{u_{n}\right\}$ which is nonnegative and strictly subnormal for $n \geq N$.

Proof. If $0 \leq w_{n}<1$ for $n \geq N-\tau^{*}$, then the companion sequence $\left\{w^{(t)}\right\}$ of (3.1) satisfies

$$
w_{n}^{(0)}=0 \leq w_{n}<1, \quad n \geq N-\tau^{*} .
$$

Therefore,

$$
\frac{1}{\left(1-w_{s}^{(0)}\right)^{\alpha_{i j}}} \leq \frac{1}{\left(1-w_{s}\right)^{\alpha_{i j}}}, \quad n \geq N-\tau^{*}
$$

so that

$$
\begin{aligned}
w_{n}^{(0)}=0 \leq w_{n}^{(1)}= & b(n) w_{n-\sigma}^{(0)} \prod_{i=n-\sigma}^{n-1} \frac{1}{1-w_{i}^{(0)}} \\
& +\sum_{i=1}^{k} p_{i}(n) \prod_{j=1}^{m_{i}} \prod_{s=n-\tau_{i j}(n)}^{n-1} \frac{1}{\left(1-w_{s}^{(0)}\right)^{\alpha_{i j}}} \\
\leq & b(n) w_{n-\sigma} \prod_{i=n-\sigma}^{n-1} \frac{1}{1-w_{i}} \\
& +\sum_{i=1}^{k} p_{i}(n) \prod_{j=1}^{m_{i}} \prod_{s=n-\tau_{i j}(n)}^{n-1} \frac{1}{\left(1-w_{s}\right)^{\alpha_{i j}}} \leq w_{n}
\end{aligned}
$$


for $n \geq N$. Inductively, we may show that $w_{n}^{(0)} \leq w_{n}^{(1)} \leq w_{n}^{(2)} \leq \ldots \leq w_{n}<1$ for $n \geq N$.

Conversely, if each $w^{(t)}$ is strictly subnormal and $\left\{w^{(t)}\right\}$ is pointwise convergent to a sequence $\left\{u_{n}\right\}$ which is nonnegative and strictly subnormal for $n \geq N$, then as before, we may show that $w_{n}^{(0)} \leq w_{n}^{(1)} \leq w_{n}^{(2)} \leq \ldots \leq$ $u_{n}<1$ for $n \geq N$. By the Lebesgue dominated convergence theorem, we may take limits on both sides of (3.4) as $t$ tends to infinity to obtain

$$
u_{n}=b(n) u_{n-\sigma} \prod_{i=n-\sigma}^{n-1} \frac{1}{\left(1-u_{i}\right)}+\sum_{i=1}^{k} p_{i}(n) \prod_{j=1}^{m_{i}} \prod_{s=n-\tau_{i j}(n)}^{n-1} \frac{1}{\left(1-u_{s}\right)^{\alpha_{i j}}}
$$

for $n \geq N$. The proof is complete.

We pause to make two important observations here. Note first that the companion sequence $\left\{w^{(t)}\right\}$ is nondecreasing in $t$ (so that $0 \leq w_{n}^{(t)} \leq w_{n}^{(t+1)}$ for $n \geq N$ ) provided each $w^{(t)}$ is strictly subnormal. We will refer to this property as the monotone nature of the companion sequence $\left\{w^{(t)}\right\}$. Note further that the proof of Theorem 3.2.2 shows that $\left\{u_{n}\right\}$ satisfies (3.6) for $n \geq N$, which is a system of equalities (cf. (3.2)). Since we may easily verify that the sequence $\left\{x_{n}\right\}$ defined by $x_{N}=1$ and (3.3) is an eventually positive solution of

$$
\begin{aligned}
& \Delta x_{n}-b(n) \Delta x_{n-\sigma} \\
& \quad+\sum_{i=1}^{k} p_{i}(n) \prod_{j=1}^{m_{i}}\left|x_{n-\tau_{i j}(n)}\right|^{\alpha_{i j}} \operatorname{sgn} x_{n-\tau_{i j}(n)}=0, \quad n \geq 0,
\end{aligned}
$$

and since every solution of (3.7) is also a solution of (3.1), we see that the following result must hold.

THEOREM 3.2.3. The relation (3.1) has an eventually positive nonincreasing solution if, and only if, (3.7) has an eventually positive nonincreasing solution.

In case $b(n) \equiv b, p_{i}(n) \equiv p_{i}>0$ for $1 \leq i \leq k$, and $\tau_{i j}(n) \equiv \tau_{i j}$ for $1 \leq i \leq k$ and $1 \leq j \leq m_{i}$, the relation (3.1) reduces to

$$
\Delta x_{n}-b \Delta x_{n-\sigma}+\sum_{i=1}^{k} p_{i} \prod_{j=1}^{m_{i}}\left|x_{n-\tau_{i j}}\right|^{\alpha_{i j}} \operatorname{sgn} x_{n-\tau_{i j}} \leq 0, \quad n \geq 0,
$$

and for large $N$, the sequence $\left\{w_{n}^{(0)}\right\}_{n=N}^{\infty}$ becomes the constant sequence $\{0\}$, and the sequence $\left\{w_{n}^{(1)}\right\}_{n=N}^{\infty}$ becomes the constant sequence $\left\{w^{(1)}\right\}$ defined by $w^{(1)}=\lambda_{1}$, where

$$
\lambda_{1}=\sum_{i=1}^{k} p_{i}>0
$$


Inductively, we see that if we define $\lambda_{0}=0$ and

$$
\lambda_{t+1}=b \frac{\lambda_{t}}{\left(1-\lambda_{t}\right)^{\sigma}}+\sum_{i=1}^{k} p_{i}\left(1-\lambda_{t}\right)^{-\sum_{j=1}^{m_{i}} \alpha_{i j} \tau_{i j}}, \quad t=0,1,2, \ldots,
$$

then $\left\{w_{n}^{(t)}\right\}$ is the constant sequence $\left\{\lambda_{t}\right\}$ for each $t \geq 1$. If (3.8) has an eventually positive and nonincreasing solution, then by Theorem 3.2.2, $\lambda_{t}<1$ for $t=0,1,2, \ldots, 0<\lambda_{1} \leq \lambda_{2} \leq \ldots<1$, and $\left\{\lambda_{t}\right\}$ converges to a number in $(0,1)$. The converse clearly holds.

THEOREM 3.2.4. The relation (3.8) has an eventually positive nonincreasing solution if, and only if, the sequence $\left\{\lambda_{t}\right\}$ defined by $\lambda_{0}=0$ and (3.9) satisfies

$$
0=\lambda_{0} \leq \lambda_{1} \leq \lambda_{2} \leq \ldots<1
$$

and converges to a number in $(0,1)$.

Therefore if (3.8) has an eventually positive nonincreasing solution, then by taking limits on both sides of (3.9), we see that the associated "characteristic equation"

$$
\lambda=\frac{b \lambda}{(1-\lambda)^{\sigma}}+\sum_{i=1}^{k} p_{i}(1-\lambda)^{-\sum_{j=1}^{m_{i}} \alpha_{i j} \tau_{i j}}
$$

has a real root $\lambda=\lambda^{*}$ in $(0,1)$. Conversely, if (3.10) has a real root $\lambda=\lambda^{*}$ in $(0,1)$, then

$$
0<\lambda_{1}=\sum_{i=1}^{k} p_{i} \leq \sum_{i=1}^{k} p_{i}\left(1-\lambda^{*}\right)^{-\sum_{j=1}^{m_{i}} \alpha_{i j} \tau_{i j}} \leq \lambda^{*},
$$

and inductively, $\lambda_{t} \leq \lambda^{*}$ for all $t \geq 2$. By the monotone nature of the sequence $\left\{w^{(t)}\right\}$, we see that $\left\{\lambda_{t}\right\}$ is also monotone and hence it converges to a number in $\left(0, \lambda^{*}\right]$. Thus, $(3.8)$ has an eventually positive nonincreasing solution by Theorem 3.2.2.

To summarize, (3.8) has an eventually positive nonincreasing solution if, and only if, the characteristic equation $(3.10)$ has a real root in $(0,1)$.

We remark that if we assume that $p_{i}=0$ for $1 \leq i \leq k$ in the above theorem, the assertion remains true if we change $(0,1)$ to $[0,1)$. Indeed, the corresponding $\left\{\lambda_{t}\right\} \equiv\{0\}$ and the corresponding relation is

$$
\Delta x_{n}-b \Delta x_{n-\sigma} \leq 0, \quad n \geq 0,
$$

which has the nonnegative and nonincreasing solution $\{1\}$.

3.3. Consequences. As an immediate corollary of Theorem 3.2.1, we seek a constant solution $\{w\}$ of (3.2) and come up with the following result. 
TheOREM 3.3.1. If there is a number $w \in[0,1)$ and an integer $N \geq \tau^{*}$ such that

$$
w \geq \frac{b(n) w}{(1-w)^{\sigma}}+\sum_{i=1}^{k} p_{i}(n)(1-w)^{-\sum_{j=1}^{m_{i}} \alpha_{i j} \tau_{i j}(n)}, \quad n \geq N,
$$

then (3.1) has an eventually positive nonincreasing solution.

Therefore, if $\sigma=1, b(n) \equiv 1 / 2$, and

$$
\sum_{j=1}^{m_{i}} \alpha_{i j} \tau_{i j}(n) \equiv \tau>1, \quad 1 \leq i \leq k, n \geq 0,
$$

then (3.1) will have an eventually positive nonincreasing solution provided that

$$
\sum_{i=1}^{k} p_{i}(n) \leq \frac{w^{*}\left(1-2 w^{*}\right)\left(1-w^{*}\right)^{\tau-1}}{2}, \quad n \geq N,
$$

where $w^{*}=1-\sqrt{1-1 /(2(1+\tau))}$. Indeed, (3.11) will be satisfied if $\sum_{i=1}^{k} p_{i}(n) \leq w(1-w)^{\tau}-\frac{b w(1-w)^{\tau}}{(1-w)^{\sigma}}=\frac{w(1-2 w)(1-w)^{\tau-1}}{2}, \quad 0 \leq w<1$, which in turn will be satisfied if

$$
\sum_{i=1}^{k} p_{i}(n) \leq \max _{0 \leq w<1} \frac{w(1-2 w)(1-w)^{\tau-1}}{2},
$$

where the positive maximum is attained at $w=w^{*}$, which is a positive and strictly subnormal constant solution of (3.11).

As an immediate corollary of Theorems 3.2.1 and 3.2.2, we have the following result.

THEOREM 3.3.2. The relation (3.1) cannot have an eventually positive and nonincreasing solution if for any integer $N \geq \tau^{*}$, there is some integer $n \geq N$ such that $w_{n}^{(t)} \geq 1$ for some $t \geq 0$.

Therefore, if for any $N \geq \tau^{*}$, there is some integer $n \geq N$ such that

$$
w_{n}^{(1)}=\sum_{i=1}^{k} p_{i}(n) \geq 1,
$$

then (3.1) cannot have an eventually positive nonincreasing solution.

THEOREM 3.3.3. Suppose there is a constant $\tau$ such that

$$
0 \leq \sum_{j=1}^{m_{i}} \alpha_{i j} \tau_{i j}(n) \leq \tau, \quad n \geq N-\tau^{*} \geq 0,1 \leq i \leq k .
$$


Suppose further that

$$
\mu_{1}=\inf _{n \geq N-\tau^{*}} \sum_{i=1}^{k} p_{i}(n)>0
$$

and

$$
\inf _{n \geq N-\tau^{*}, 0<\mu<1} \frac{1}{\mu}\left\{\frac{b(n) \mu}{(1-\mu)^{\sigma}}+\sum_{i=1}^{k} p_{i}(n)(1-\mu)^{-\sum_{j=1}^{m_{i}} \alpha_{i j} \tau_{i j}(n)}\right\}>1 .
$$

Then (3.1) cannot have an eventually positive nonincreasing solution.

Proof. Let $\left\{w^{(t)}\right\}$ be the companion sequence of (3.1). In view of Theorem 3.3.2, we may assume without loss of generality that each $w^{(t)}$ is nonnegative and is subnormal for $n \geq N$. Let

$$
\mu_{1}=\inf _{n \geq N-\tau^{*}} w_{n}^{(1)}=\inf _{n \geq N-\tau^{*}} \sum_{i=1}^{k} p_{i}(n) \geq 0,
$$

where $N-\tau^{*} \geq 0$. Then

$$
\begin{aligned}
w_{n}^{(2)} & =b(n) w_{n-\sigma}^{(1)} \prod_{i=n-\sigma}^{n-1} \frac{1}{1-w_{i}^{(1)}}+\sum_{i=1}^{k} p_{i}(n) \prod_{j=1}^{m_{i}} \prod_{s=n-\tau_{i j}(n)}^{n-1} \frac{1}{\left(1-w_{s}^{(1)}\right)^{\alpha_{i j}}} \\
& \geq \frac{b(n) \mu_{1}}{\left(1-\mu_{1}\right)^{\sigma}}+\sum_{i=1}^{k} p_{i}(n)\left(1-\mu_{1}\right)^{-\sum_{j=1}^{m_{i}} \alpha_{i j} \tau_{i j}(n)}
\end{aligned}
$$

for $n \geq N$, and

$$
w_{n}^{(2)} \geq \mu_{2}
$$

for $n \geq N-\tau^{*}$, where

$$
\mu_{2}=\inf _{n \geq N-\tau^{*}}\left\{\frac{b(n) \mu_{1}}{\left(1-\mu_{1}\right)^{\sigma}}+\sum_{i=1}^{k} p_{i}(n)\left(1-\mu_{1}\right)^{-\sum_{j=1}^{m_{i}} \alpha_{i j} \tau_{i j}(n)}\right\} .
$$

Inductively, if we define

$$
\mu_{t+1}=\inf _{n \geq N-\tau^{*}}\left\{\frac{b(n) \mu_{t}}{\left(1-\mu_{t}\right)^{\sigma}}+\sum_{i=1}^{k} p_{i}(n)\left(1-\mu_{t}\right)^{-\sum_{j=1}^{m_{i}} \alpha_{i j} \tau_{i j}(n)}\right\}
$$

for $t=0,1, \ldots$, then

$$
w_{n}^{(t)} \geq \mu_{t}, \quad n \geq N-\tau^{*}, t \geq 1
$$

In view of (3.15), if $\mu_{t} \geq 1$ for any $t \geq 1$, then our assertion is true. Assume that $\mu_{t}<1$ for $t \geq 1$. We assert that $0<\mu_{1} \leq \mu_{2} \leq \ldots<1$. Indeed, $\mu_{1}>0$ 
by assumption. Thus

$$
\begin{aligned}
\mu_{2} & =\inf _{n \geq N-\tau^{*}}\left\{\frac{b(n) \mu_{1}}{\left(1-\mu_{1}\right)^{\sigma}}+\sum_{i=1}^{k} p_{i}(n)\left(1-\mu_{1}\right)^{-\sum_{j=1}^{m_{i}} \alpha_{i j} \tau_{i j}(n)}\right\} \\
& \geq \inf _{n \geq N-\tau^{*}} \sum_{i=1}^{k} p_{i}(n)=\mu_{1} .
\end{aligned}
$$

Inductively, we see that $0<\mu_{1} \leq \mu_{2} \leq \ldots<1$ as required. Let $\mu^{*}=$ $\lim _{t \rightarrow \infty} \mu_{t}$. If $\mu^{*}<1$, then in view of $(3.14)$, we have

$$
\mu^{*}=\inf _{n \geq N-\tau^{*}}\left\{\frac{b(n) \mu^{*}}{\left(1-\mu^{*}\right)^{\sigma}}+\sum_{i=1}^{k} p_{i}(n)\left(1-\mu^{*}\right)^{-\sum_{j=1}^{m_{i}} \alpha_{i j} \tau_{i j}(n)}\right\}
$$

contrary to $(3.13)$. Hence $\mu^{*}=1$. But then by means of (3.15), we see that $w_{n}^{(\infty)} \geq 1$. This shows, in view of Theorem 3.2.2, that (3.1) cannot have an eventually positive nonincreasing solution. The proof is complete.

Note that for each fixed $n \geq N$,

$$
\inf _{0<\mu<1}\left\{\frac{1}{\mu} p_{i}(n)(1-\mu)^{-\sum_{j=1}^{m_{i}} \alpha_{i j} \tau_{i j}(n)}\right\}
$$

is attained at

$$
\mu_{i}=\left(1+\sum_{j=1}^{m_{i}} \alpha_{i j} \tau_{i j}(n)\right)^{-1}
$$

and is equal to

$$
\frac{p_{i}(n)\left(1+\tau_{i}(n)\right)^{1+\tau_{i}(n)}}{\left(\tau_{i}(n)\right)^{\tau_{i}(n)}}
$$

where

$$
\tau_{i}(n)=\sum_{j=1}^{m_{i}} \alpha_{i j} \tau_{i j}(n)<\infty, \quad 1 \leq i \leq k .
$$

Note further that for each fixed $n \geq N$,

$$
\inf _{0<\mu<1} \frac{b(n)}{(1-\mu)^{\sigma}}
$$

is attained at $b(n)$. Thus, if

$$
\liminf _{n \rightarrow \infty}\left\{b(n)+\sum_{i=1}^{k} \frac{p_{i}(n)\left(1+\tau_{i}(n)\right)^{1+\tau_{i}(n)}}{\left(\tau_{i}(n)\right)^{\tau_{i}(n)}}\right\}>1,
$$

then, by Theorem 3.3.3, (3.1) cannot have an eventually positive nonincreasing solution. 
Next, note that the arithmetic mean-geometric mean inequality implies

$$
\begin{aligned}
\frac{1}{\mu} \sum_{i=1}^{k} p_{i}(n)(1-\mu)^{-\tau_{i}(n)} & \geq \frac{k}{\mu}\left\{\prod_{i=1}^{k} p_{i}(n)\right\}^{1 / k}\left\{\prod_{i=1}^{k}(1-\mu)^{-\tau_{i}(n)}\right\}^{1 / k} \\
& =k\left\{\prod_{i=1}^{k} p_{i}(n)\right\}^{1 / k}\left\{\prod_{i=1}^{k} \frac{1}{\mu(1-\mu)^{\tau_{i}(n)}}\right\}^{1 / k},
\end{aligned}
$$

where $\tau_{i}(n)$ is defined by (3.16). Since

$$
\inf _{0<\mu<1} \frac{1}{\mu(1-\mu)^{\tau_{i}(n)}}=\frac{\left(\tau_{i}(n)+1\right)^{\tau_{i}(n)+1}}{\tau_{i}(n)^{\tau_{i}(n)}},
$$

it follows that if $p_{1}(n)+\ldots+p_{k}(n)$ does not vanish identically for all large $n$ and

$$
\liminf _{n \rightarrow \infty}\left\{b(n)+k\left[\prod_{i=1}^{k} p_{i}(n)\right]^{1 / k}\left[\prod_{i=1}^{k} \frac{\left(\tau_{i}(n)+1\right)^{\tau_{i}(n)+1}}{\tau_{i}(n)^{\tau_{i}(n)}}\right]^{1 / k}\right\}>1,
$$

then, by Theorem 3.3.3, (3.1) cannot have an eventually positive nonincreasing solution.

THEOREM 3.3.4. Suppose there is a constant $\psi \in(0,1)$ such that

$$
\sup _{n \geq N-\tau^{*} \geq 0} \frac{1}{\psi}\left\{\frac{b(n) \psi}{(1-\psi)^{\sigma}}+\sum_{i=1}^{k} p_{i}(n)(1-\psi)^{-\sum_{j=1}^{m_{i}} \alpha_{i j} \tau_{i j}(n)}\right\} \leq 1 .
$$

Then (3.1) has an eventually positive nonincreasing solution.

Proof. Let $\left\{w^{(t)}\right\}$ be the companion sequence of (3.1). Let

$$
\psi_{1}=\sup _{n \geq N-\tau^{*}} w_{n}^{(1)}=\sup _{n \geq N-\tau^{*}} \sum_{i=1}^{k} p_{i}(n) \geq 0,
$$

where $N-\tau^{*} \geq 0$. By (3.18), $0 \leq \psi_{1} \leq \psi$. Next,

$$
\begin{aligned}
w_{n}^{(2)} & =b(n) w_{n-\sigma}^{(1)} \prod_{i=n-\sigma}^{n-1} \frac{1}{1-w_{i}^{(1)}}+\sum_{i=1}^{k} p_{i}(n) \prod_{j=1}^{m_{i}} \prod_{s=n-\tau_{i j}(n)}^{n-1} \frac{1}{\left(1-w_{s}^{(1)}\right)^{\alpha_{i j}}} \\
& \leq \frac{b(n) \psi_{1}}{\left(1-\psi_{1}\right)^{\sigma}}+\sum_{i=1}^{k} p_{i}(n)\left(1-\psi_{1}\right)^{-\sum_{j=1}^{m_{i}} \alpha_{i j} \tau_{i j}(n)}
\end{aligned}
$$

for $n \geq N$ and

$$
w_{n}^{(2)} \leq \psi_{2}
$$

for $n \geq N-\tau^{*}$, where

$$
\psi_{2}=\sup _{n \geq N-\tau^{*}}\left\{\frac{b(n) \psi_{1}}{\left(1-\psi_{1}\right)^{\sigma}}+\sum_{i=1}^{k} p_{i}(n)\left(1-\psi_{1}\right)^{-\sum_{j=1}^{m_{i}} \alpha_{i j} \tau_{i j}(n)}\right\} .
$$


Inductively, if we define

$$
\begin{aligned}
& \psi_{t+1} \\
= & \sup _{n \geq N-\tau^{*}}\left\{\frac{b(n) \psi_{t}}{\left(1-\psi_{t}\right)^{\sigma}}+\sum_{i=1}^{k} p_{i}(n)\left(1-\psi_{t}\right)^{-\sum_{j=1}^{m_{i}} \alpha_{i j} \tau_{i j}(n)}\right\}, \quad t \geq 0,
\end{aligned}
$$

then

$$
w_{n}^{(t)} \leq \psi_{t}, \quad n \geq N-\tau^{*}, t \geq 1
$$

Under the condition (3.18), it is easily verified that $0 \leq \psi_{1} \leq \psi_{2} \leq \ldots \leq \psi$. Thus, in view of (3.20), we see that the companion sequence $\left\{w^{(t)}\right\}$ satisfies $w_{n}^{(t)} \leq \psi_{t} \leq \psi$, which implies $w_{n}^{(\infty)} \leq \psi<1$ for $n \geq N$. The proof is complete.

As further applications of the previous results, we will derive a comparison theorem for the relation (3.1). Let us consider a majorant inequality of the form

$$
\begin{aligned}
& \Delta y_{n}-B(n) \Delta y_{n-\xi} \\
& \quad+\sum_{i=1}^{k} P_{i}(n) \prod_{j=1}^{m_{i}}\left|y_{n-\sigma_{i j}(n)}\right|^{\alpha_{i j}} \operatorname{sgn} y_{n-\sigma_{i j}(n)} \leq 0, \quad n \geq 0,
\end{aligned}
$$

where $\{B(n)\}_{n=0}^{\infty}$ is a real sequence, $\left\{P_{1}(n)\right\}_{n=0}^{\infty}, \ldots,\left\{P_{k}(n)\right\}_{n=0}^{\infty}$ are nonnegative sequences, $\xi$ is a positive integer, and $\sigma_{11}(n), \ldots, \sigma_{k m_{k}}(n)$ are nonnegative integers which satisfy conditions similar to those satisfied by $\tau_{i j}(n)$, and the other parameters or parameter sequences are the same as those in (3.1). Also let the sequence $\left\{W^{(t)}\right\}$ of sequences be the companion sequence of (3.21) relative to a large integer $n: W_{n}^{(0)}=0$ for $n \geq 0$; and for $t=0,1,2, \ldots$,

$W_{n}^{(t+1)}=B(n) W_{n-\xi}^{(t)} \prod_{i=n-\xi}^{n-1} \frac{1}{1-W_{i}^{(t)}}+\sum_{i=1}^{k} P_{i}(n) \prod_{j=1}^{m_{i}} \prod_{s=n-\sigma_{i j}(n)}^{n-1} \frac{1}{\left(1-W_{s}^{(t)}\right)^{\alpha_{i j}}}$

for $n \geq N$ and $W_{n}^{(t+1)}=0$ for $n<N$.

TheOREM 3.3.5. Suppose $\sigma \leq \xi, b(n) \leq B(n), \tau_{i j}(n) \leq \sigma_{i j}(n)$ for $n \geq 0$ and $p_{i}(n) \leq P_{i}(n)$ for $1 \leq i \leq \bar{k}, 1 \leq j \leq m_{k}$ and $n \geq 0$. If (3.21) has an eventually positive nonincreasing solution, then so does (3.1).

Indeed, if (3.21) has an eventually positive solution, then the relation

$$
W_{n} \geq B(n) W_{n-\xi} \prod_{t=n-\xi}^{n-1} \frac{1}{1-W_{t}}+\sum_{i=1}^{k} P_{i}(n) \prod_{j=1}^{m_{i}} \prod_{s=n-\sigma_{i j}(n)}^{n-1} \frac{1}{\left(1-W_{s}\right)^{\alpha_{i j}}}
$$


has an eventually strictly subnormal and eventually nonnegative solution $\left\{W_{n}\right\}$. Thus

$$
W_{n} \geq b(n) W_{n-\sigma} \prod_{t=n-\sigma}^{n-1} \frac{1}{1-W_{t}}+\sum_{i=1}^{k} p_{i}(n) \prod_{j=1}^{m_{i}} \prod_{s=n-\tau_{i j}(n)}^{n-1} \frac{1}{\left(1-W_{s}\right)^{\alpha_{i j}}},
$$

which shows that $\left\{W_{n}\right\}$ is also an eventually subnormal and eventually nonnegative solution of (3.2). Our assertion now follows from Theorem 3.2.1.

\section{References}

[1] R. P. Agarwal, Difference Equations and Inequalities, Marcel Dekker, New York, 1992.

[2] I. Györi and G. Ladas, Oscillation Theory of Delay Differential Equations, Clarendon Press, Oxford, 1991.

[3] I. Györi and M. Pituk, Asymptotic formulae for the solutions of a linear delay difference equation, J. Math. Anal. Appl. 195 (1995), 376-392.

[4] B. S. Lalli, Oscillation theorems for neutral difference equations, Comput. Math. Appl. 28 (1994), 191-202.

[5] B. S. Lalli, B. G. Zhang and J. Z. Li, On the oscillation of solutions and existence of positive solutions of neutral difference equations, J. Math. Anal. Appl. 158 (1991), 213-233.

[6] J. Popenda, The oscillation of solutions of difference equations, Comput. Math. Appl. 28 (1994), 271-279.

[7] J. Yan, Oscillation of solutions of first order delay differential equations, Nonlinear Anal. 11 (1987), 1279-1287.

[8] J. Yan and C. Qian, Oscillation and comparison results for delay difference equations, J. Math. Anal. Appl. 165 (1992), 346-360.

[9] J. S. Y u, A note on "Oscillation of solutions of first order delay differential equations", J. Math. Res. Exposition 10 (1990), 290-293.

[10] B. G. Zhang and S. S. Cheng, Oscillation criteria and comparison theorems for delay difference equations, Fasc. Math. 25 (1995), 13-32.

Department of Mathematics

Tsing Hua University

Hsinchu, Taiwan 30043, R.O.C.

E-mail: sscheng@math2.math.nthu.edu.tw
Department of Mathematics Datong Advanced College Datong, Shanxi 037008, P.R. China 